\title{
Review: Autophagy and Immune Senescence
}

Hanling Zhang, Daniel J. Puleston, Anna Katharina Simon*

Kennedy Institute of Rheumatology, Oxford University, Roosevelt Drive, Oxford OX3 7FY, Oxfordshire, UNITED KINGDOM

*Correspondence to: Katja Simon katja.simon@kennedy.ox.ac.uk 


\begin{abstract}
With extension of the average lifespan, ageing has become a heavy burden in society. Immune senescence is a key risk factor for many age-related diseases such as cancer and increased infections in the elderly, and hence, has elicited much attention in recent years. As our body's guardian, the immune system maintains systemic health through removal of pathogens and damage. Autophagy is an important cellular "clearance" process by which a cell internally delivers damaged organelles and macromolecules to lysosomes for degradation. Here, we discuss the most current knowledge of how impaired autophagy can lead to cellular and immune senescence. We also provide an overview, with examples, of the clinical potential of exploiting autophagy to delay immune senescence and/or rejuvenate immunity to treat various age-related diseases.
\end{abstract}

\title{
Autophagy: A Key Mechanism Controlling Immune Senescence
}

Autophagy is a highly conserved catabolic cellular process in which cells deliver their own components to lysosomes for degradation. There are three types of autophagy: macroautophagy, microautophagy, and chaperone-mediated autophagy. Macroautophagy (hereafter referred to as autophagy), so far the most thoroughly studied type of autophagy, involves the formation of doublemembrane autophagosomes that carry bulk cellular materials to lysosomes (Box 2). Through specific degradation of damaged organelles, protein aggregates, and intracellular pathogens, it has recently come to light that autophagy is a key quality control mechanism for cellular homeostasis, and with age, is coupled to cellular senescence. Indeed, autophagy is impaired with age in different cell types, tissues and organisms.

Immune senescence refers to a series of age-associated deteriorative changes in the development and functions of the innate and adaptive immune system. This causes inefficient control of infections and tissue damage. Impaired immune surveillance with age also increases the risk of tumorigenesis. Recent findings suggest that autophagy deficient immune cells display features of premature ageing while aged macrophages and T cells have decreased autophagy $[1,2]$. Interestingly, treatments inducing autophagy are able to improve $\mathrm{T}$ cell and $\mathrm{B}$ cell responses against infections [1,3]. In this review, we first discuss mechanisms of autophagy control of cellular senescence. Second, we examine the mechanisms of immune senescence with age, as well as the clinical potential of modulating autophagy to prevent or treat immune senescence-related diseases. 


\section{Autophagy Controls Cellular Senescence}

Impaired autophagy during ageing has been reported in various mammalian tissues such as human brain [4] and murine T cells and macrophages [1,2]. Multiple autophagy signaling pathways and the lysosomal compartment are affected with age (Figure 1), and may together contribute to inefficient autophagy induction and degradation (Box 3). There are also other changes in aged cells including accumulation of damaged organelles and protein aggregates, oxidative stress, DNA mutations and telomere dysfunctions. These changes are linked with each other and with autophagy in a complex manner to cause cellular dysfunction and senescence, which can lead to systemic inflammation and organismal ageing (Figure 2). Below, we review evidence on different mechanisms by which autophagy might control ageing at the cellular level.

\section{Mitochondria and ROS}

Ageing is associated with the accumulation of damaged mitochondria, that are less efficient in ATP generation and produce more ROS [5]. The elevated ROS further oxidize cellular components including proteins, lipids, DNA, and RNA to cause oxidative stress $[6,7]$. Autophagy controls mitochondrial health through degradation of damaged mitochondria (mitophagy). Autophagy-deficient yeast accumulates dysfunctional mitochondria and ROS [8], while autophagy induction reduces ROS and improves cell survival [9] (Figure 2).

However, the contribution of ROS to ageing has become controversial in recent years and may depend on the dose. Rather than simply being a 'troublemaker', ROS also provide stress signals that initiate anti-ageing responses. For example, catalase inactivation or calorie restriction in yeast extends their chronological lifespan by elevating $\mathrm{H}_{2} \mathrm{O}_{2}$ levels, which activates superoxide dismutases and reduces oxidative damage of macromolecules [10]. Mutations in mitochondrial superoxide dismutase sod-2 or in mitochondrial respiratory chain components, as well as treatment with the pro-oxidant paraquat, elevates ROS levels and extends the lifespan of worms, despite an increase in oxidatively-damaged proteins $[11,12]$. The induced ROS are interpreted as an anti-ageing signal because in this setting, antioxidants such as N-acetylcysteine (NAC) or vitamin C, suppress the lifespan extension effect [12]. In reality, ROS can also activate autophagy through both transcriptional and post-transcriptional mechanisms [13], which may in turn, limit the oxidative damage progression.

Therefore, ROS can directly cause cellular damage, while also activating stress responses such as autophagy. If these responses are efficiently activated to prevent further ROS accumulation and oxidative damage, cellular homeostasis can be maintained. Otherwise, the continued accumulation of ROS and damage may eventually negatively tip the balance and lead to cellular senescence or death.

\section{Genome stability}

Autophagy indirectly maintains genomic stability through limiting ROS accumulation (Figure 2). The somatic mutation theory of ageing proposes that 
DNA mutations accumulate with age to cause functional decline and senescence in somatic cells [14]. ROS attack single nucleobases to induce DNA point mutations, strand breaks, as well as inter- and intra-strand crosslinks [15]. The mitochondrial protectant $\alpha$-LA and other antioxidants are able to reduce chromosomal breaks in lymphocytes from Fanconi anemia patients [16], suggesting a causal role of ROS in genomic instability. Telomeres are also vulnerable sites of DNA damage, and a large number of DNA damage foci accumulate at telomeres [17]. Thus ROS-induced damage may cause telomere dysfunction to further deteriorate genome stability.

\section{Proteostasis}

Cells tightly control their protein homeostasis (proteostasis) in two ways, namely, chaperone-assisted protein folding and protein degradation. When unfolded proteins cannot refold in time, they are tagged with polyubiquitin for proteasome- or autophagy-mediated degradation. While the proteasome mainly degrades K48-polyubiquitinated single proteins, autophagy tends to degrade K63-polyubiquitinated protein aggregates and organelles. Large-scale protein aggregation is found in aged mice [18], indicating the disruption of proteostasis with ageing. Indeed, both systems of protein folding and protein degradation are impaired with age [19]. Moroever, increased ROS levels further intensify oxidative protein damage and cause protein aggregation [18]. Therefore, autophagy can control proteostasis by direct clearance of protein aggregates and also, by preventing the formation of protein aggregates when limiting ROS accumulation (Figure 2).

\section{Inflammation}

Mammalian ageing is associated with a chronic inflammation status, named inflammaging [20]. Autophagy positively or negatively regulates inflammation in different settings (Figure 2). Autophagy (xenophagy) delivers cytosolic and endosomal PAMPs to lysosomes for TLR7 and TLR9 recognition to trigger innate immune responses, and pharmaceutical or genetic inhibition of autophagy has been shown to block the detection of PAMPs and subsequent TLR activation in murine dendritic cells and B cells $[21,22]$. The autophagy machinery is also used for secretion of proteins including inflammatory cytokines [23]. Instead of degrading the cargo, the lysosome assists in the activation and secretion of cytokines such as IL-1 $\beta$ ashown in primary murine bone marrow-derived macrophages [23].

Autophagy can also suppress inflammation by directly degrading the ubiquitinated inflammasome [24], and inhibiting its activation by clearing intracellular damage. For example, inhibition of autophagy in cultured human cells causes increased inflammasome activation [25], and mice with autophagy deficiency in myeloid cells show spontaneous lung inflammation [26]. It has been proposed that this phenomenon can be explained, at least in part, by a loss in mitochondrial integrity, because mitochondrial DNA (mtDNA) and ROS released from damaged mitochondria are able to trigger inflammasome activation in murine macrophages [27]. Moreover, protein aggregates folded as amyloid fibrils, which are potential autophagy substrates, are also shown to stimulate TLR2 signaling and IL-1 $\beta$ production [28]. 
Autophagy most likely plays an overall suppressive role in inflammation in the context of inflammaging. Deficient autophagy causes accumulation of cellular damage and intracellular infectious particles, which further triggers inflammation signaling. When excessive cellular damage accumulates, cells will enter senescence, further acquiring a senescence-associated secretory phenotype and reinforcing inflammation [29].

\section{Immune Senescence}

The overall features of innate immune senescence are chronic activation, decreased responsiveness to stimulation, reduced antigen processing and presentation. Adaptive immune senescence is associated with loss of TCR and BCR repertoire diversity, impaired effector responses against new antigens and impaired memory formation. While autophagy is not involved in all of these processes, it is required for both innate and adaptive immune functions, and autophagy-deficient immune cells display premature ageing. In the next section we will discuss in detail how mammalian immune cells change during ageing and the relationship between autophagy and immune senescence.

\section{Innate Immunity}

\section{Monocytes/Macrophages}

The overall number of circulating monocytes remains constant with age in human, but the $\mathrm{CD} 14^{+} \mathrm{CD} 16^{+}$inflammatory monocytes are reported increased [30], suggesting a chronic activation of monocytes in the elderly. Similarly, there is an increase of tissue-resident inflammatory macrophages in aged mice [31]. Indeed, aged mice present a bias towards harboring more pro-inflammatory M1like macrophages, as opposed to tissue-repairing anti-inflammatory M2-like macrophages [32]. Consistently, macrophages from aged mice display a higher basal level of inflammasome activation and IL-1 $\beta$ and IL-18 production, which are associated with increased ROS [33]. However aged macrophages down regulate TLR expression [34, 35], and are less responsive to antigen stimulation $[35,36]$ (Table 1).

Autophagy is required for monocyte-macrophage differentiation and their activation [37]. Moreover, macrophages from mice with early (during differentiation) Atg 7 deletion display reduced expression of activation surface markers such as MHC I/II and CD86 [2] (Table 1). This could be due to either a block in macrophage activation, and/or to delivery defects in activation markers to the cell surface. Autophagy deficiency also leads to reduced nitric oxide (NO) production [2], deficient phagocytosis [37] and impaired bactericidal ability in macrophages [2]. Mitochondria and ROS have been found to accumulate in autophagy-deficient macrophages [2], resulting in increased production of proinflammatory cytokines such as IL-1 $\beta$ [38]. Furthermore, a later knockout of Atg 5 in differentiated macrophages has been reported to enhance M1 macrophage polarization in vivo, causing systemic inflammation, which is a typical ageing-phenotype (inflammaging) [39]. Indeed aged macrophages present reduced autophagic flux [2], which together with the observations of 
multiple ageing-like phenotypes of autophagy-deficient macrophages, suggests that impaired autophagy contributes to macrophage dysfunction during ageing.

\section{Dendritic cells}

The number of both plasmacytoid and myeloid DCs decreases during ageing in humans and mice $[40,41]$. Similar to aged macrophages and neutrophils, aged DCs also show a number of functional defects in migration, phagocytosis, and antigen presentation (Table 1).

Upon infection, autophagy is induced by activated NODs and TLRs in DCs, which is required for multiple responses $[42,43]$. The induced autophagy delivers cytosolic as well as endocytosed extracellular antigens to lysosomes for further TLRs activation, cytokine secretion, and antigen presentation [21, 44] (Table 1). Defective autophagy may cause DC ageing via the loss of quality control of mitochondria and ROS. A higher ROS level is found in aged murine plasmacytoid DCs, and the reduction of ROS by antioxidant treatments or calorie restriction partly recovers their IFN- $\alpha$ production in response to TLR9 stimulation [45]. This raises the possibility of improving the functions of DCs in the elderly via autophagy induction, and potentially ameliorating adaptive immunity through enhanced antigen presentation.

\section{Neutrophils}

Neutrophils are numerous and short-lived granulocytes that quickly respond to injuries and infections. Following chemokine gradients, neutrophils migrate to sites of inflammation and control damage through phagocytosis, degranulation and, although controversial, neutrophil extracellular traps (NETs) formation. The number of peripheral neutrophils remains relatively constant during ageing in human [46], but neutrophils from elderly humans show decreased phagocytosis of bacteria and fail to kill intracellular bacteria, suggesting defects in endocytosis and xenophagy [47]. Zhang and colleagues also reported that aged murine neutrophils are in an overall active state [48]. Chronic stimulation from the microbiota and infections can cause over activation and tissue accumulation of neutrophils in aged mice, which further contribute to excessive inflammation and tissue damage [49]. In contrast to the increased basal inflammation, the chemotactic and infiltration properties of murine neutrophils are impaired with age when chemokine levels are kept constant [50]. Upon cutaneous Staphylococcus aureus wound infection, circulating neutrophils in aged mice show elevated chemokine receptor CXCR2 expression but decreased adhesion molecule ICAM-1 expression [50], indicating that signal transduction downstream of CXCR2 might be blocked in neutrophils during ageing. The cytotoxicity of neutrophils is also impaired with age in both mice and humans, including reduced superoxide burst, lysosomal degranulation, and the formation of NETs in response to stimulation $[47,51,52]$.

Most studies suggest that optimal activation of both human and murine neutrophils requires autophagy. Firstly, autophagy is induced upon human neutrophil activation [53], and secondly autophagy deficiency causes a series of functional defects, including reduced degranulation in murine neutrophils [54], decreased killing of intracellular pathogens in human neutrophils and reduced 
NET formation although the latter was only shown with PI3K inhibitors that may also affect NET formation itself [55], [56] (Table 1). Considering that aged neutrophils are less functional and autophagy is possibly required for optimal neutrophil activation, it would be interesting to see if autophagy is impaired in aged neutrophils and if autophagy induction improves aged neutrophil functions.

\section{Adaptive Immunity \\ T cells}

Of all age-associated alterations in the immune system, the T cell compartment is the most affected by the passage of time. Involution of the thymus results in a severe drop in the output of naive T cells. The lack of new T cell specificities emanating from the thymus, coupled to increased homeostatic proliferation, results in contraction of the peripheral TCR repertoire [57], severely impacting the ability of the $\mathrm{T}$ cell pool to respond to infections such as influenza in aged mice [58]. TCR repertoire restriction in the aged can also be driven by clonal expansion, which in clinically healthy humans is often a consequence of infection with chronic viruses such as cytomegalovirus (CMV) that drive out growth of certain $\mathrm{CD}^{+} \mathrm{T}$ cell clones [59]. Expansion of CMV-specific CD8 ${ }^{+} \mathrm{T}$ cells can come to dominate the peripheral repertoire, with up to $23 \%$ of all $\mathrm{CD}^{+} \mathrm{T}$ cells specific for a single CMV epitope in some instances [59].

Cumulative exposure to foreign pathogens throughout life leads to a preponderance of activated T cells (also called virtual memory) over naïve cells during the latter stages of life. Both $\mathrm{CD}^{+}$and $\mathrm{CD} 8^{+} \mathrm{T}$ cells undergo similar phenotypic shifts; however, the rate at which these occur and accumulate differs. In humans, however, the relative proportions of memory subsets remain reasonably stable in $\mathrm{CD}^{+} \mathrm{T}$ cells, while for $\mathrm{CD} 8^{+} \mathrm{T}$ cells, the ageing central memory compartment contracts by as much as $50 \%$ with a corresponding increase in terminally differentiated effector cells [60,61]. Amongst CD4 ${ }^{+} \mathrm{T}$ cells, Th1 cells exhibit a gradual decline in numbers with old age, followed later by Th2 cells [62], whilst CD4+ FoxP3+ Tregs increase in number [63].

Accompanying these phenotypic changes are deleterious changes in $\mathrm{T}$ cell function. Both $\mathrm{CD}^{+}{ }^{+}$and $\mathrm{CD} 8^{+} \mathrm{T}$ cell effector responses are defective in response to infections with reduced expression of key inflammatory mediators such as IFN- $\gamma$, TNF- $\alpha$, IL-2 and granzyme B in aged mice $[64,65]$. Poor proliferation and diminished responsiveness to IL-2 appears to be a ubiquitous feature of aged $\mathrm{T}$ cells and might explain the delayed peak in T cell expansion and cytotoxic activity commonly observed in the elderly. For instance, Almanzar and colleagues show that CMV-specific $\mathrm{CD}^{+}{ }^{+} \mathrm{CD} 28^{-}$effector $\mathrm{T}$ cells in the elderly fail to proliferate ex vivo in response to antigenic challenge, even in the presence of exogenous IL-2 [60]. The reduced expression of key inflammatory mediators such as IFN- $\gamma$, TNF- $\alpha$, and granzyme B are all suggestive of decreased effector capacity in aged $\mathrm{T}$ cells $[64,65]$. In $\mathrm{CD} 4^{+} \mathrm{T}$ cells, the loss of CD28 also leads to a concomitant defect in CD40L expression and along with it the capacity to help B cell proliferation and antibody production [66]. 
The formation of T cell memory, and therefore long-term immunity, is also perturbed in old age. Memory T cells are able to persist for long-periods after initial infection and provide rapid, protective responses to a second infection with the same pathogen. Defective secondary expansion of CD8 ${ }^{+} \mathrm{T}$ cells during recall responses to virus infections such as lymphocytic choriomeningitis virus [67] and influenza [1] have been observed in aged mice. Interestingly, memory $\mathrm{CD}^{+}{ }^{+} \mathrm{T}$ cells formed in young TCR transgenic mice have been found to function normally well into old age, in contrast to memory cells formed in aged mice which respond poorly to secondary exposure to cognate antigen [68]. This suggests that the age of a naïve $\mathrm{T}$ cell when it first encounters antigen may be a crucial determinant of effective T cell memory. In the case of Tregs in elderly individuals, a mixed picture emerges. There are reports that the suppressive capacity of Tregs is not deleteriously affected in old age [69]. However, other studies document a reduced ability of Tregs in aged mice to suppress syngeneic $\mathrm{CD}^{+} \mathrm{T}$ cell proliferation relative to allogeneic $\mathrm{CD} 4^{+} \mathrm{T}$ cells [70] and to suppress IL-17-producing CD4 ${ }^{+} \mathrm{T}$ cells [71]. These findings imply a potential defect of aged Tregs to control autoimmune proliferation, consistent with increased susceptibility to autoimmunity in elderly patients.

Despite normal proliferation of effector cells, the formation and maintenance of memory CD8 ${ }^{+} \mathrm{T}$ cells are severely impaired in vivo in the absence of autophagy $[1,72]$, similar to what is observed during ageing. Also reminiscent of aged phenotypes, cytokine production [73] and proliferation [73-75] is impaired in activated autophagy-deficient murine T cells ex vivo. Autophagy-deficient T cells contain expanded mitochondria [76] and ER [77], as well as increased ROS [76], indicating that autophagy maintains organelle homeostasis to sustain long-term survival. Furthermore, ATP generation of ex vivo activated murine Th1 cells has been shown to be decreased when autophagy is blocked by lysosomal inhibitors $\mathrm{NH}_{4} \mathrm{Cl}$ and leupeptin, but giving methyl pyruvate as an exogenous energy source partially rescues this defect [73]. When senescence of human $\mathrm{CD}^{+} \mathrm{T}$ cells is reversed by blocking p38 signaling, autophagy also provides the energy [78]. Interestingly, work from our laboratory has shown that autophagy levels are reduced in $\mathrm{CD}^{+}{ }^{+} \mathrm{T}$ cells from aged mice [1] and humans [79]. The autophagyinducing compound spermidine [80] can restore aged CD8 ${ }^{+} \mathrm{T}$ cell effector and memory responses to influenza-infected mice back to "young"-like levels [1]. The mTOR-dependent autophagy inducer rapamycin has also been shown to improve memory T cell responses against LCMV infection in mice and vaccinia virus Ankara in primates [81]. Therefore, strategies to increase autophagy levels in aged mice could be beneficial when attempting to improve vaccination approaches in the elderly. However, further investigations are required to determine the potential of other autophagy-inducing compounds in improving immune memory, as well as the mechanisms underlying various putative beneficial effects of autophagy.

Recent findings also show that murine T cell-specific deletion of Atg7, Atg5, Atg1611, or Vps34 impairs the survival of Tregs [82-84], which leads to chronic intestinal inflammation [84]. It is possible that $\mathrm{T}$ cell regulation will be boosted by autophagy induction, which may also help control the systemic chronic inflammation in the elderly. 


\section{B cells}

The production rate of bone marrow pre-B cells and peripheral mature B cells is lower in aged mice relative to young, leading to a slower turnover of peripheral B cell pools [85]. The number of peripheral B cells is well maintained or slightly decreased in aged mice due to clonal expansion of chronically activated B cells in response to environmental antigens [86, 87]. However naïve B cells decline with age, and the peripheral B-cell receptor (BCR) repertoire diversity decreases [88].

In elderly human subjects, yellow fever virus or influenza vaccination studies show that antibody responses are delayed, or quantitatively reduced, respectively within 28 days post vaccination $[89,90]$. Indeed, germinal center

reactions have been shown to be impaired in aged human B cells [91]. Declined functions of T cells and DCs are key factors leading to compromised B cell activation [92], while ex vivo experiments also show impaired up-regulation of the enzyme AID in response to influenza vaccine in B cells from elderly humans [93]. These findings thus indicate both extrinsic and intrinsic activation defects of aged B cells. Crucial players in most vaccinations are long-lived class-switched plasma cells and memory B cells. Consequently, it may be possible that fewer class-switched plasma cells and memory B cells form due to a decline in the germinal center reaction with age. However, whether these newly formed cells also exhibit long-term survival or re-activation defects requires further investigation.

Autophagy is required for both B cell development and function. Murine knockouts of essential autophagy genes such as Atg 5 lead to decreased numbers of pre-B cells and newly formed peripheral B cells [94]. Autophagy is induced during murine plasma cell differentiation [95]. Murine plasma cells without Atg5 show increased ER stress and cell death, failing to give rise to an efficient antibody response and to form long-lived plasma cells in vivo [95], indicating that autophagy is essential for ER quality control in these professional antibodysecreting cells. Both human and murine memory B cells present constitutively high autophagy levels, a requirement for their long-term survival [3]. Mice with Atg 7 knockout in B cells generate comparable memory B cells 2 weeks after immunization with NP-KLH antigen, but undergo more cell death in week 8 [96], indicating that $\mathrm{B}$ cell autophagy is dispensable for the initial formation but essential for long-term maintenance of memory B cells. Moreover, autophagy inducer rapamycin further enhances murine memory B cells survival and their responses against influenza infection [3]. Although warranting validation, ongoing studies are investigating a role for impaired autophagy in aged B cells to determine if there is indeed a causal role for autophagy deficiency in compromised $\mathrm{B}$ cell responses with age.

In contrast to conventional B cells (B-2 B cells), B-1 B cells possess innate immune functions. This self-renewing population produced from bone marrow progenitors secretes polyspecific autoantibodies against self- and bacterial antigens such as phosphatidylcholine, and does not form memory [97]. Both the number of B-1 B cells and the production of autoantibodies have been found to significantly increase in aged mice [86]. However knockout of essential 
autophagy genes Atg 5 or Atg 7 has been reported to lead to decreased formation of murine B-1 B cells $[3,94,95]$. Both ageing of B-1 B cells and the link to with autophagy still requires further investigation.

\section{Autophagy as a Target to Treat Age-related Disorders}

\section{Infections and vaccination}

The aged population is highly susceptible to infection. In the USA, more than $90 \%$ of 36,000 influenza-related annual deaths occur in individuals aged 65 years or over [98]. Vaccination offers one of our best hopes in removing the burden of infectious diseases. However, poor vaccine efficacy in the elderly only complicates matters [99]. Consequently, it is important to begin incorporating our knowledge of immune senescence into vaccine designs against diseases that represent a particular burden for the aged. In this regard, promising strategies have included increasing antigen dosage delivered in vaccine preparations. For instance, a high dose of influenza vaccine was found to improve immune responses in the elderly, although protection rates remained lower than in young individuals receiving regular, lower doses [100]. Adjuvant administration represents another way to efficiently boost immune responses to vaccination. And, autophagy inducers such as rapamycin and spermidine, improve memory $\mathrm{T}$ cell $[1,81]$ and B cell responses [3]. This raises the possibility of following these mechanisms and inducing autophagy to potentially improve vaccine efficacy. Indeed, more autophagy inducer candidates could be screened for their use as immune-boosting adjuvants. Meanwhile a better mechanistic understanding of autophagy in immune senescence may facilitate the identification of more specific targets for adjuvant design (see Clinician's corner).

On the other hand, Lu and colleagues reported that the elevated lung inflammation caused by deletion of autophagy gene Epg5, Atg7, Atg5, Atg14, and Fip200 in myeloid cells protects mice from influenza infection [101]. HubbardLucey and colleagues also reported that deletion of autophagy gene Atg16l1 enhances inflammatory signaling of DC [102]. These observations suggest the caveat that autophagy induction may limit basal inflammation in myeloid cells in the young and thus dampen immune responses. Multiple aspects of innate immunity including phagocytosis, inflammation, and interactions with adaptive immunity might be differentially affected by autophagy, which requires a comprehensive evaluation in both young and aged settings.

\section{Tumor immunity}

The risk of malignancy significantly increases with age due to the accumulation of tumorigenic mutations and concomitant loss of immune surveillance. Aged DCs have been reported to be less potent in stimulating tumor specific CD8+ $\mathrm{T}$ cell responses that restrict tumor growth in murine melanoma models [103]. Moreover, with regard to aged T cell immunity, both contracted TCR repertoires and defective effector/memory responses may contribute to impaired tumor surveillance and clearance. Therefore, interventions to delay or rejuvenate 
immune senescence may be helpful in lowering the risk of tumorigenesis and progression. Indeed, immune cells can be manipulated to obtain a more robust response against tumors, which is the principle of tumor immunotherapy [104]. Consequently, autophagy induction might be able to render a beneficial effect on generating tumor-specific adaptive and innate immunity, providing a promising clinical potential to improve immunotherapeutic efficacy.

\section{Atherosclerosis}

Endothelial oxidized low-density lipoprotein deposits initiate the pathogenesis of atherosclerosis. Macrophages can function to ingest these lipid deposits and prevent disease aggravation. However, excess lipid ingestion imposes a heavy burden on macrophage lipid metabolism, and failure to efficiently remove accumulated intracellular lipids leads to the formation of foam cells and eventually, cell death. Autophagy (lipophagy) degrades lipid droplets in foam cells to mobilize them for cholesterol efflux, which promotes the reverse cholesterol transport back to the liver for intestinal excretion [105]. Inhibitors of mTOR such as rapamycin and simvastatin, have been shown to prevent atherosclerosis in mammalian models like rabbits [106]. Although these mTORinhibitors are functionally pleiotropic, they potentially raise an opportunity to induce autophagy in macrophages to prevent or treat atherosclerosis.

The above listed disorders are just three examples illustrating how autophagy has become, by potentially rejuvenating immune responses, a novel drug target. Other examples include age-related diseases caused by chronic inflammation.

\section{Concluding Remarks}

Immune senescence leads to inefficient removal of foreign pathogens and local damage, which in turn results in multiple age-related diseases. The immune system consists of both short-lived cells (e.g. myeloid cells) and long-lived cells (e.g. memory T and B cells). Immune senescence refers to changes in all immune subpopulations during ageing, but we would argue that the cellular mechanisms are different. It is conceivable that functional changes in short-lived cells result from extrinsic factors such as inflammation as well as intrinsic factors such as inherited damage from stem cells and stem cell progenitors. Cell divisions during differentiation may help to "dilute" cellular damage. However, for these cells, autophagy may contribute to remodeling processes during differentiation and activation. By contrast, long-lived (and often quiescent) cells have a higher chance of acquiring and accumulating cellular damage. Consequently, they rely heavily on autophagy to maintain homeostasis, exhibiting a severe survival defect in the absence of autophagy.

Some autophagy inducers such as rapamycin and spermidine have been shown to provide anti-ageing effects across species, while particularly promoting adaptive immunity. Meanwhile, the aged innate immune system is hyperinflammatory but less responsive to infections and damages, which is consistent with the phenotypes of autophagy deficiency. Further investigations are 
warranted to establish whether autophagy induction alleviates overall inflammation in the elderly and improves or reduces innate immunity. However, autophagy inducers available to date do not not work efficiently in primary mammalian cells, revealing the need to undertake drug screening in specific primary cell-based systems. Although many limitations and unknowns remain (Outstanding Questions), our current knowledge raises the possibility that increasing the autophagy process may delay or rejuvenate immune senescence, which in turn will prevent or alleviate age-related diseases and increase the health span of our ageing population.

\section{Acknowledgements}

We apologize to all scientists whose important contributions were not cited due to space limitations. The Chinese Scholarship Council is gratefully acknowledged for the stipend and fees of $\mathrm{HZ}$ and the Wellcome Trust for providing general support with an investigator award. We thank the National Institute of Health Research (NIHR) for providing the salary of DP through the Oxford Biomedical Research Center (BRC). 


\section{Box 1. Clinician's Corner box}

- While the senescent innate immune system is characterized by chronic inflammation, it is less responsive to simulation, has reduced bactericidal activity and antigen presentation.

- The senescent adaptive immune system has a restricted TCR/BCR repertoire diversity. Aged T cells and B cells show delayed and decreased responses to new antigens and are less able to form efficient immunological memory, an important reason for the compromised vaccination efficacy in the elderly.

- Immune senescence contributes to the pathogenesis of many age-related diseases including cancer, atherosclerosis, increased susceptibility to infection, and also, overall inflammation.

- Autophagy levels decline with age, likely contributing to immune senescence.

- Several autophagy inducers and autophagy-inducing interventions such as rapamycin, spermidine and fasting have been shown to improve adaptive immunity and harbor a protective effect in atherosclerosis. Considering the pleiotropic functions of these existing compounds, specific, mTOR-independent autophagy inducers are yet to be identified. 


\section{Box 2. The Process of Autophagy}

- Upon autophagy induction, the activated ULK1 protein kinase complex forms proximally to the ER, mitochondria or other membrane structures [107].

- The class III PI3-kinase complex is recruited by the ULK1 complex, producing phosphatidylinositol 3-phosphate (PI(3)P) which drives the formation of the isolation membrane (autophagophore).

- The autophagophore expands to engulf cytoplasmic materials including organelles and soluble components. This expansion requires two ubiquitinlike reactions, ATG12-ATG5-ATG16L and LC3-phosphatidylethanolamine (LC3-PE).

- ATG12 is activated by ATG7 (E1-like) and then transferred to ATG5 by ATG10 (E2-like); ATG12-ATG5 forms a complex with ATG16L.

- LC3 is activated by ATG7 (E1-like) and conjugated to PE by ATG3 (E2 like). Then ATG12-ATG5-ATG16L complex functions as an E3-like ligase to add the phosphatidylethanolamine tail for LC3 lipidation.

- Finally, the elongated membrane closes to form mature autophagosomes, which fuse with lysosomes to begin degradation.

Starvation induced bulk autophagy degradation is thought to be non-selective, whereas selective autophagy controls the quality of cytoplasmic constituents. The specific recognition of damaged organelles and protein aggregates is mediated by autophagy adaptors such as p62, NBR1 and NDP52. These adaptors recognize polyubiquitin chains on damaged proteins and organelles, and interact with autophagy proteins such as ULK1 [108] and LC3 to initiate autophagy and guide the engulfment process. 


\section{Box 3. Age-related changes in pathways linked to autophagy}

A variety of signaling pathways upstream of autophagy change with age, however, the effect on autophagy is largely unknown.

When environmental nutrients are sufficient, the conserved insulin/IGF-1 signaling pathway is activated and turns off autophagy via activation of mTORC1 and inhibition of FOXO transcription factors [109] (Figure 1. The suppression of the insulin/IGF-1 signaling pathway by prolonged fasting rejuvenates the haematopoietic and immune system in aged mice [111]. ). mTORC1 itself also senses intracellular amino acids, and inhibits autophagy induction via phosphorylation of ULK1, a protein essential for autophagy initiation, and the master regulator TFEB [110] (Figure 1)mTORC1 is overactivated in aged tissues such as HSCs [112], and genetic or pharmacological inhibition of mTORC1 signaling has been found to rejuvenate HSC functions in aged mice [112]. These findings suggest a causal role of excessive mTORC1 signaling in haematopoietic ageing. In addition, activated mTORC1 may impose a suppressive signal on autophagy induction during ageing.

Nutrient starvation is usually associated with a shortage of energy supply. AMPK (AMP-activated protein kinase) senses the energy crisis and turns on autophagy directly through ULK1 and class III PI3K $[113,114]$ and indirectly, by inhibiting mTORC1 (Figure 1). Overexpression and activation of AMPK have been found to induce autophagy and prolong the lifespan across species [115]. AMPK also enhances SIRT1 activity by increasing cellular NAD+ levels [116] (Figure 1). Mammalian SIRT1 is a NAD ${ }^{+}$-dependent deacetylase that regulates a wide range of cellular processes involving metabolism, inflammation, apoptosis and consequently ageing. SIRT1 activates autophagy through deacetylation of autophagy-related proteins including ATG5, ATG7 and LC3 [117], and autophagy regulators such as AMPK [118]. Overexpression or activation of SIRT1 extends lifespan across species including mice in an autophagy-dependent manner [119]. Consistent with decreased AMPK expression and activation found in myocardial tissues of aged mice [120], both intracellular NAD ${ }^{+}$levels and SIRT1 activity decline in multiple organs of aged rats [121]. Therefore, decreased activity of AMPK-SIRT1 may desensitize cells to age-related stress signals and lead to inefficient autophagy induction.

Autophagy delivers material for degradation to the lysosome (Figure 1), an organelle, which is also affected during ageing in several systems. The acidity of lysosome-like vacuoles in yeast declines during asymmetric divisions, and preventing this decline by overexpressing the $\mathrm{H}^{+}$-ATPase VMA1 improves mitochondrial health and extends lifespan [122]. The age-pigment lipofuscin that consists of non-degradable oxidized aggregates accumulates in lysosomes with age. Lipofuscin decreases lysosomal enzyme activity [124] and impairs autophagy induction [125] as shown in cultured human cells. Therefore the lysosome is an interesting target to increase autophagic efficiency. However, 
lysosomal activity in aged mammalian tissues remains largely unexplored, and efficient ways to improve lysosomal function are to be developed.

\section{Glossary}

AID: Activation-induced cytidine deaminase is the enzyme that deaminates cytosine bases. It is involved in somatic hypermutation, class-switch recombination, and gene conversion for antibody diversification.

Central memory T cells: Memory T cells with a capacity for cell renewal that circulate throughout the blood and lymphatic system and confer long-term protection against bacteria, viruses and other pathogens.

Dendritic cells (DCs): Professional antigen-presenting cells in the mammalian immune system, also producing cytokines in response to infections. The two main subsets of DCs in human and mice are Myeloid DCs are CD11 $\mathrm{c}^{+}$and display conventional functions of DCs including antigen-presentation and cytokine production. Plasmacytoid DCs are CD11 $\mathrm{c}^{-}$and are professional type I IFNproducing cells, which are also present antigens for T cell activation although less efficient than myeloid DCs.

Fanconi anemia: A rare genetic disease associated with mutations in DNA repair genes. The development of certain cancers such as acute myelogenous leukemia is a hallmark, as is bone marrow failure in affected individuals.

Foam cells: Macrophages with accumulated lipid droplets giving them a foamy cytoplasm.

FOXOs: Forkhead box 0 proteins are transcription factors suppressed by Insulin/IGF-1 signaling. They regulate many important stress-related cellular processes including cell cycle arrest, apoptosis, metabolism and autophagy.

Germinal Center Reaction: A process where antigen-specific B cells expand and undergo class switching and somatic hypermutation to form high-affinity plasma cells and memory B cells.

Insulin/IGF-1 signaling pathway: Insulin-like growth factor (IGF-1) is a hormone that is produced by the liver and other tissues in response to growth hormone stimulation. Insulin and IGF-1 share intracellular pathways including AKT-mTOR and small GTPase Ras to promote cell growth.

Monocytes/Macrophages: Differentiated from monocytes, macrophages are professional phagocytes that engulf dying cells, damaged or foreign substances. They also produce cytokines and present antigens to T cells. There are two subtypes of macrophages: M1 macrophages are pro-inflammatory with a strong 
microbicidal activity, while $\mathbf{M} 2$ macrophages decrease inflammation and promote tissue repair.

mTORC1: The mammalian target of rapamycin complex 1 is composed of the catalytic subunit mTOR and regulatory proteins including RAPTOR, mLST8, DEPTOR, and PRAS40. Regulated by amino acids levels and signals from AKT and AMPK, mTORC1 promotes cellular anabolism like protein translation and suppresses autophagy.

Neutrophil extracellular traps (NETs): NETs are extracellular fibril structures released by activated neutrophils. They consist of granule proteins and chromatin and can trap and kill pathogens. The in vivo function of NETs is still unclear.

PAMPs: Pathogen-associated molecular patterns are molecular motifs that are specific to individual or groups of pathogens that bind to pattern recognition receptors such as TLRs and NODs expressed by cells of the innate immune system facilitating the identification of non-self.

TFEB: Transcription factor EB is a basic helix-loop-helix protein recognizing Ebox (5'-CANNTG-3') and more specifically CLEAR-box (5'-GTCACGTGAC- 3'). Lysosomal and autophagy genes are targets of TFEB, so TFEB promotes autophagosome and lysosome biogenesis.

Th1 cells: A class of CD4+ $\mathrm{T}$ helper cells defined by the expression of the transcription factor T-bet and the inflammatory mediator IFN- $\gamma$ that is typically important for macrophage activation and protection against intracellular pathogens.

Th2 cells: A subset of $C D 4^{+} \mathrm{T}$ helper cells defined by the expression of the transcription factor GATA-3 and the cytokines IL-4 and IL-13. Th2 cells are typically important for the activation of other immune cells such as eosinophils, basophils and mast cells and like other helper subsets are important for B cell activation and antibody production.

Tregs: A type of suppressive T cell that limits inflammation through the expression of inhibitory receptors and soluble anti-inflammatory cytokines such as IL-10 and TGF- $\beta$. 
Table 1. Effects of Ageing and Autophagy Deficiency on Innate Immunity (- Unchanged; $\downarrow$ Decreased; $\uparrow$ Increased)

\begin{tabular}{|c|c|c|c|c|}
\hline & Phenotypes & Ageing & $\begin{array}{l}\text { Autophagy } \\
\text { deficiency }\end{array}$ & Causal link \\
\hline \multirow[t]{10}{*}{$\begin{array}{l}\text { Macrophage } \\
\text { S }\end{array}$} & $\begin{array}{l}\text { Peripheral number of } \\
\text { monocytes/macrophag } \\
\text { es }\end{array}$ & $\begin{array}{l}\text { Circulating } \\
\text { monocytes - } \\
\text { Inflammator } \\
\text { y monocytes } \\
\& \text { Tissue } \\
\text { macrophage } \\
\mathrm{s} \uparrow[30,31 \text {, } \\
126]\end{array}$ & $\uparrow[2]$ & \multirow[t]{10}{*}{$\begin{array}{l}\text { Autophagy } \\
\text { is } \\
\text { decreased } \\
\text { in aged } \\
\text { macrophag } \\
\text { es [2]. }\end{array}$} \\
\hline & $\begin{array}{l}\text { Monocyte-macrophage } \\
\text { differentiation }\end{array}$ & $-[126]$ & $\begin{array}{l}\downarrow[37] \text { or - } \\
{[2]}\end{array}$ & \\
\hline & $\begin{array}{l}\text { Polarization towards } \\
\text { M1macrophage } \\
\text { subtype }\end{array}$ & $\uparrow[32]$ & $\uparrow[39]$ & \\
\hline & Basal inflammation & $\uparrow[33]$ & $\begin{array}{l}\uparrow[2,38, \\
101]\end{array}$ & \\
\hline & Mitochondria integrity & $\downarrow[33]$ & $\downarrow[2,38]$ & \\
\hline & $\begin{array}{l}\text { TLR expression and } \\
\text { response to TLR } \\
\text { stimulation }\end{array}$ & $\downarrow[34,35]$ & $\begin{array}{l}\text { TLR4 } \downarrow \text { but } \\
\text { cytokine } \\
\text { productio } \\
\mathrm{n} \uparrow \text { in } \\
\text { response } \\
\text { to LPS [2] }\end{array}$ & \\
\hline & $\begin{array}{l}\text { Surface marker } \\
\text { expression }\end{array}$ & $\begin{array}{l}\downarrow[127] \text { or - } \\
{[103]} \\
\text { (MHC II, } \\
\text { CD80, CD86) }\end{array}$ & $\begin{array}{l}\downarrow(\mathrm{MHC} \\
\mathrm{I} / \mathrm{II}, \mathrm{CD} 86) \\
{[2]}\end{array}$ & \\
\hline & $\begin{array}{l}\text { Antigen presentation } \\
\text { and T cell activation }\end{array}$ & $\downarrow[36]$ & $\downarrow[128]$ & \\
\hline & Phagocytosis & $\begin{array}{l}\downarrow[129] \text { or }- \\
{[103]}\end{array}$ & $\downarrow[2,37]$ & \\
\hline & NO production & $\downarrow[35]$ & $\downarrow[2]$ & \\
\hline \multirow{5}{*}{$\begin{array}{l}\text { Dendritic } \\
\text { cells }\end{array}$} & Peripheral DC number & $\downarrow[40,41]$ & $-[44]$ & \multirow{5}{*}{$\begin{array}{l}\text { Higher ROS } \\
\text { in aged } \\
\text { pDCs } \\
\text { causes } \\
\text { impaired } \\
\text { IFN- } \alpha \\
\text { production } \\
\text { [45]. }\end{array}$} \\
\hline & Chemotaxis & $\downarrow[103,130]$ & $-[131]$ & \\
\hline & Phagocytosis & $\begin{array}{l}\downarrow[130] \text { or }- \\
{[103]}\end{array}$ & - [131] & \\
\hline & $\begin{array}{l}\text { Lysosomal antigen } \\
\text { detection }\end{array}$ & $?$ & $\downarrow[21]$ & \\
\hline & $\begin{array}{l}\text { Surface marker } \\
\text { expression }\end{array}$ & $\begin{array}{l}\downarrow \text { (MHC- } \\
\text { peptide } \\
\text { complexes, } \\
\text { CD40, CD86, } \\
\text { TLRs) [40] }\end{array}$ & $\begin{array}{l}\downarrow \text { (MHC II) } \\
{[132],-} \\
\text { (MHC II, } \\
\text { CD40, } \\
\text { CD86) }\end{array}$ & \\
\hline
\end{tabular}




\begin{tabular}{|c|c|c|c|c|}
\hline & & & $\begin{array}{l}{[131] \text { or } \uparrow} \\
\text { (CD40, } \\
\text { CD80, } \\
\text { CD86) } \\
{[102]}\end{array}$ & \\
\hline & $\begin{array}{l}\text { Antigen presentation } \\
\text { and } T \text { cell activation }\end{array}$ & $\downarrow[103]$ & $\begin{array}{l}\downarrow[44,131, \\
132] \text { or } \uparrow \\
{[102]}\end{array}$ & \\
\hline \multirow[t]{6}{*}{ Neutrophils } & Chemotaxis & $\begin{array}{l}\downarrow[47,50, \\
51]\end{array}$ & $?$ & \multirow[t]{6}{*}{ NA } \\
\hline & Phagocytosis & $\downarrow[47]$ & $\downarrow[56]$ & \\
\hline & Superoxide burst & - or $\downarrow[47]$ & $\downarrow[54]$ & \\
\hline & Mitochondrial integrity & $\downarrow[51]$ & $?$ & \\
\hline & $\begin{array}{l}\text { Lysosomal } \\
\text { degranulation }\end{array}$ & $\downarrow[52]$ & $\downarrow[54]$ & \\
\hline & NET formation & $\downarrow[51]$ & $\downarrow[55]$ & \\
\hline
\end{tabular}


Table 2. Effects of Ageing and Autophagy Deficiency on Adaptive immunity (- Unchanged; $\downarrow$ Decreased; $\uparrow$ Increased)

\begin{tabular}{|c|c|c|c|c|}
\hline & Phenotypes & Ageing & $\begin{array}{l}\text { Autophagy } \\
\text { deficiency }\end{array}$ & Causal link \\
\hline \multirow{8}{*}{$\mathrm{T}$ cells } & $\begin{array}{l}\text { Formation of Naïve } \\
\text { T cells }\end{array}$ & $\begin{array}{l}\downarrow \text { (Thymus } \\
\text { involution) }\end{array}$ & $\begin{array}{l}\downarrow \text { (Defective } \\
\text { survival) [1, } \\
75]\end{array}$ & \multirow{8}{*}{$\begin{array}{l}\text { 1. Reduced } \\
\text { autophagy in } \\
\text { aged CD8 }{ }^{+} \mathrm{T} \\
\text { cells }[1,79] \\
\text { 2. Autophagy- } \\
\text { inducing } \\
\text { compound } \\
\text { spermidine } \\
\text { rejuvenates } \\
\text { aged CD8 }{ }^{+} \mathrm{T} \\
\text { cell responses } \\
\text { [1]. } \\
\text { 3. Autophagy } \\
\text { provides } \\
\text { energy for } \\
\text { reversing the } \\
\text { senescence of } \\
\text { EMRA CD8 }{ }^{+} \mathrm{T} \\
\text { cells [78]. }\end{array}$} \\
\hline & $\begin{array}{l}\text { TCR repertoire } \\
\text { diversity }\end{array}$ & $\downarrow[57]$ & $?$ & \\
\hline & $\begin{array}{l}\text { Ratio of activated } \\
\text { cells to naïve cells }\end{array}$ & $\uparrow$ & $\uparrow[1]$ & \\
\hline & \begin{tabular}{|l} 
Cytokine production \\
\end{tabular} & $\downarrow[64,65]$ & $\downarrow[73]$ & \\
\hline & Help for B cells & $\downarrow[66]$ & $?$ & \\
\hline & Proliferation & $\downarrow[60]$ & $\begin{array}{l}\downarrow \text { (ex vivo })[73- \\
75] \text { or }-(\text { in } \\
\text { vivo })[1,72]\end{array}$ & \\
\hline & $\begin{array}{l}\text { Formation and } \\
\text { maintenance of } \\
\text { memory T cells }\end{array}$ & $\downarrow[1,67,68]$ & $\downarrow[1,72]$ & \\
\hline & $\begin{array}{l}\text { Treg } \\
\text { function/survival }\end{array}$ & $\begin{array}{l}\downarrow[70,71] \text { or }- \\
{[69]}\end{array}$ & $\downarrow[82-84]$ & \\
\hline \multirow{6}{*}{ B cells } & $\begin{array}{l}\text { Number of pre-B } \\
\text { cells and newly } \\
\text { formed mature B } \\
\text { cells }\end{array}$ & $\downarrow[85]$ & $\downarrow[94]$ & \multirow[t]{6}{*}{$\begin{array}{l}\text { Reduced } \\
\text { autophagy in } \\
\text { aged B cells }\end{array}$} \\
\hline & $\begin{array}{l}\text { BCR repertoire } \\
\text { diversity }\end{array}$ & $\downarrow[88]$ & $?$ & \\
\hline & $\begin{array}{l}\text { Germinal centre } \\
\text { reaction }\end{array}$ & $\downarrow[91,93]$ & - $[95,96]$ & \\
\hline & Humoral response & $\begin{array}{l}\downarrow \text { (delayed or } \\
\text { reduced) [89, } \\
90]\end{array}$ & $\downarrow[95]$ & \\
\hline & $\begin{array}{l}\text { Long-term } \\
\text { maintenance of } \\
\text { newly formed } \\
\text { memory }\end{array}$ & $?$ & $\begin{array}{l}\downarrow \text { (Both long- } \\
\text { lived plasma } \\
\text { cells [95] and } \\
\text { memory B } \\
\text { cells [3]) }\end{array}$ & \\
\hline & $\begin{array}{l}\text { B-1 population and } \\
\text { autoantibody } \\
\text { production }\end{array}$ & $\uparrow[86]$ & $\downarrow[3,94,95]$ & \\
\hline
\end{tabular}




\section{References}

1 Puleston, D.J., et al. (2014) Autophagy is a critical regulator of memory CD8(+) T cell formation. eLife 3

2 Stranks, A.J., et al. (2015) Autophagy Controls Acquisition of Aging Features in Macrophages. Journal of innate immunity 7, 375-391

3 Chen, M., et al. (2014) Essential role for autophagy in the maintenance of immunological memory against influenza infection. Nature medicine 20, 503-510 4 Rubinsztein, D.C., et al. (2011) Autophagy and aging. Cell 146, 682-695 5 Sohal, R.S. and Sohal, B.H. (1991) Hydrogen peroxide release by mitochondria increases during aging. Mechanisms of ageing and development 57, 187-202 6 Grimm, S., et al. (2011) Protein oxidative modifications in the ageing brain: consequence for the onset of neurodegenerative disease. Free radical research 45 , 73-88

7 Nie, B., et al. (2013) Age-dependent accumulation of 8-oxoguanine in the DNA and RNA in various rat tissues. Oxidative medicine and cellular longevity 2013, 303181

8 Zhang, Y., et al. (2007) The role of autophagy in mitochondria maintenance: characterization of mitochondrial functions in autophagy-deficient $\mathrm{S}$. cerevisiae strains. Autophagy 3, 337-346

9 Bin-Umer, M.A., et al. (2014) Elimination of damaged mitochondria through mitophagy reduces mitochondrial oxidative stress and increases tolerance to trichothecenes. Proceedings of the National Academy of Sciences of the United States of America 111, 11798-11803

10 Mesquita, A., et al. (2010) Caloric restriction or catalase inactivation extends yeast chronological lifespan by inducing H2O2 and superoxide dismutase activity. Proceedings of the National Academy of Sciences of the United States of America 107, 15123-15128

11 Van Raamsdonk, J.M. and Hekimi, S. (2009) Deletion of the mitochondrial superoxide dismutase sod-2 extends lifespan in Caenorhabditis elegans. PLoS genetics 5, e1000361

12 Yang, W. and Hekimi, S. (2010) A mitochondrial superoxide signal triggers increased longevity in Caenorhabditis elegans. PLoS biology 8, e1000556

13 Scherz-Shouval, R. and Elazar, Z. (2011) Regulation of autophagy by ROS: physiology and pathology. Trends in biochemical sciences 36, 30-38 14 Szilard, L. (1959) On the Nature of the Aging Process. Proceedings of the National Academy of Sciences of the United States of America 45, 30-45 15 Wang, Y. (2008) Bulky DNA lesions induced by reactive oxygen species. Chemical research in toxicology 21, 276-281

16 Ponte, F., et al. (2012) Improvement of genetic stability in lymphocytes from Fanconi anemia patients through the combined effect of alpha-lipoic acid and Nacetylcysteine. Orphanet journal of rare diseases 7, 28

17 Hewitt, G., et al. (2012) Telomeres are favoured targets of a persistent DNA damage response in ageing and stress-induced senescence. Nature communications 3,708

18 Tanase, M., et al. (2016) Role of Carbonyl Modifications on Aging-Associated Protein Aggregation. Scientific reports 6, 19311 
19 Labbadia, J. and Morimoto, R.I. (2015) The biology of proteostasis in aging and disease. Annual review of biochemistry 84, 435-464

20 Franceschi, C., et al. (2000) Inflamm-aging. An evolutionary perspective on immunosenescence. Annals of the New York Academy of Sciences 908, 244-254 21 Lee, H.K., et al. (2007) Autophagy-dependent viral recognition by plasmacytoid dendritic cells. Science 315, 1398-1401

22 Weindel, C.G., et al. (2015) B cell autophagy mediates TLR7-dependent autoimmunity and inflammation. Autophagy 11, 1010-1024

23 Dupont, N., et al. (2011) Autophagy-based unconventional secretory pathway for extracellular delivery of IL-1beta. The EMBO journal 30, 4701-4711

24 Shi, C.S., et al. (2012) Activation of autophagy by inflammatory signals limits IL-1beta production by targeting ubiquitinated inflammasomes for destruction. Nature immunology 13, 255-263

25 Shi, H., et al. (2015) Inhibition of autophagy induces IL-1beta release from ARPE-19 cells via ROS mediated NLRP3 inflammasome activation under high glucose stress. Biochemical and biophysical research communications 463, 10711076

26 Abdel Fattah, E., et al. (2015) Critical role for IL-18 in spontaneous lung inflammation caused by autophagy deficiency. Journal of immunology 194, 54075416

27 Nakahira, K., et al. (2011) Autophagy proteins regulate innate immune responses by inhibiting the release of mitochondrial DNA mediated by the NALP3 inflammasome. Nature immunology 12, 222-230

28 Gustot, A., et al. (2015) Amyloid fibrils are the molecular trigger of inflammation in Parkinson's disease. The Biochemical journal 471, 323-333 29 Zhu, Y., et al. (2014) Cellular senescence and the senescent secretory phenotype in age-related chronic diseases. Current opinion in clinical nutrition and metabolic care $17,324-328$

30 Shaw, A.C., et al. (2013) Age-dependent dysregulation of innate immunity. Nature reviews. Immunology 13, 875-887

31 Lumeng, C.N., et al. (2011) Aging is associated with an increase in T cells and inflammatory macrophages in visceral adipose tissue. Journal of immunology 187, 6208-6216

32 Gonzalez, O.A., et al. (2015) Differential Gene Expression Profiles Reflecting Macrophage Polarization in Aging and Periodontitis Gingival Tissues.

Immunological investigations 44, 643-664

33 Stout-Delgado, H.W., et al. (2016) Age-Dependent Susceptibility to Pulmonary Fibrosis is Associated with NLRP3 Inflammasome Activation. American journal of respiratory cell and molecular biology

34 Renshaw, M., et al. (2002) Cutting edge: impaired Toll-like receptor expression and function in aging. Journal of immunology 169, 4697-4701 35 Shaik-Dasthagirisaheb, Y.B., et al. (2010) Immune response of macrophages from young and aged mice to the oral pathogenic bacterium Porphyromonas gingivalis. Immunity \& ageing : I \& $A 7,15$

36 Donnini, A., et al. (2002) Phenotype, antigen-presenting capacity, and migration of antigen-presenting cells in young and old age. Experimental gerontology 37, 1097-1112 
37 Jacquel, A., et al. (2012) Autophagy is required for CSF-1-induced macrophagic differentiation and acquisition of phagocytic functions. Blood 119, 4527-4531

38 Lodder, J., et al. (2015) Macrophage autophagy protects against liver fibrosis in mice. Autophagy 11, 1280-1292

39 Liu, K., et al. (2015) Impaired macrophage autophagy increases the immune response in obese mice by promoting proinflammatory macrophage polarization. Autophagy 11, 271-284

40 Pereira, L.F., et al. (2011) Impaired in vivo CD4+ T cell expansion and differentiation in aged mice is not solely due to T cell defects: decreased stimulation by aged dendritic cells. Mechanisms of ageing and development 132, 187-194

41 Orsini, G., et al. (2012) Enumeration of human peripheral blood dendritic cells throughout the life. International immunology 24, 347-356

42 Cooney, R., et al. (2010) NOD2 stimulation induces autophagy in dendritic cells influencing bacterial handling and antigen presentation. Nature medicine 16, 90-97

43 Khan, N., et al. (2016) Signaling through NOD-2 and TLR-4 Bolsters the T cell Priming Capability of Dendritic cells by Inducing Autophagy. Scientific reports 6, 19084

44 Mintern, J.D., et al. (2015) Differential use of autophagy by primary dendritic cells specialized in cross-presentation. Autophagy 11, 906-917

45 Stout-Delgado, H.W., et al. (2008) Aging impairs IFN regulatory factor 7 upregulation in plasmacytoid dendritic cells during TLR9 activation. Journal of immunology 181, 6747-6756

46 Chatta, G.S., et al. (1994) Aging and marrow neutrophil reserves. Journal of the American Geriatrics Society 42, 77-81

47 Wenisch, C., et al. (2000) Effect of age on human neutrophil function. Journal of leukocyte biology 67, 40-45

48 Zhang, D., et al. (2015) Neutrophil ageing is regulated by the microbiome. Nature 525, 528-532

49 Stout-Delgado, H.W., et al. (2009) Aging promotes neutrophil-induced mortality by augmenting IL-17 production during viral infection. Cell host \& microbe 6, 446-456

50 Brubaker, A.L., et al. (2013) Reduced neutrophil chemotaxis and infiltration contributes to delayed resolution of cutaneous wound infection with advanced age. Journal of immunology 190, 1746-1757

51 Tseng, C.W., et al. (2012) Innate immune dysfunctions in aged mice facilitate the systemic dissemination of methicillin-resistant S. aureus. PloS one 7, e41454 52 Suzuki, K., et al. (1983) Age-related decline in lysosomal enzyme release from polymorphonuclear leukocytes after N-formyl-methionyl-leucyl-phenylalanine stimulation. Experimental hematology 11, 1005-1013

53 Mitroulis, I., et al. (2010) Regulation of the autophagic machinery in human neutrophils. European journal of immunology 40, 1461-1472

54 Bhattacharya, A., et al. (2015) Autophagy Is Required for NeutrophilMediated Inflammation. Cell reports 12, 1731-1739

55 Itakura, A. and McCarty, O.J. (2013) Pivotal role for the mTOR pathway in the formation of neutrophil extracellular traps via regulation of autophagy. American journal of physiology. Cell physiology 305, C348-354 
56 Itoh, H., et al. (2015) Enhancement of neutrophil autophagy by an IVIG preparation against multidrug-resistant bacteria as well as drug-sensitive strains. Journal of leukocyte biology 98, 107-117

57 Ahmed, M., et al. (2009) Clonal expansions and loss of receptor diversity in the naive CD8 T cell repertoire of aged mice. Journal of immunology 182, 784-792 58 Yager, E.J., et al. (2008) Age-associated decline in T cell repertoire diversity leads to holes in the repertoire and impaired immunity to influenza virus. The Journal of experimental medicine 205, 711-723

59 Khan, N., et al. (2002) Cytomegalovirus seropositivity drives the CD8 T cell repertoire toward greater clonality in healthy elderly individuals. Journal of immunology 169, 1984-1992

60 Almanzar, G., et al. (2005) Long-term cytomegalovirus infection leads to significant changes in the composition of the CD8+ T-cell repertoire, which may be the basis for an imbalance in the cytokine production profile in elderly persons. Journal of virology 79, 3675-3683

61 Czesnikiewicz-Guzik, M., et al. (2008) T cell subset-specific susceptibility to aging. Clinical immunology 127, 107-118

62 Jagger, A., et al. (2014) Regulatory T cells and the immune aging process: a mini-review. Gerontology 60, 130-137

63 Gregg, R., et al. (2005) The number of human peripheral blood CD4+ CD25high regulatory T cells increases with age. Clinical and experimental immunology 140, 540-546

64 Brien, J.D., et al. (2009) Key role of T cell defects in age-related vulnerability to West Nile virus. The Journal of experimental medicine 206, 2735-2745 65 Messaoudi, I., et al. (2006) Molecular, cellular, and antigen requirements for development of age-associated T cell clonal expansions in vivo. Journal of immunology 176, 301-308

66 Weiskopf, D., et al. (2009) The aging of the immune system. Transplant international : official journal of the European Society for Organ Transplantation $22,1041-1050$

67 Kapasi, Z.F., et al. (2002) Defective generation but normal maintenance of memory T cells in old mice. European journal of immunology 32, 1567-1573 68 Haynes, L., et al. (2003) CD4 T cell memory derived from young naive cells functions well into old age, but memory generated from aged naive cells functions poorly. Proceedings of the National Academy of Sciences of the United States of America 100, 15053-15058

69 Nishioka, T., et al. (2006) CD4+CD25+Foxp3+ T cells and CD4+CD25-Foxp3+ $\mathrm{T}$ cells in aged mice. Journal of immunology 176, 6586-6593

70 Zhao, L., et al. (2007) Changes of CD4+CD25+Foxp3+ regulatory T cells in aged Balb/c mice. Journal of leukocyte biology 81, 1386-1394

71 Sun, L., et al. (2012) Aged regulatory T cells protect from autoimmune inflammation despite reduced STAT3 activation and decreased constraint of IL17 producing T cells. Aging cell 11, 509-519

$72 \mathrm{Xu}, \mathrm{X}$. , et al. (2014) Autophagy is essential for effector CD8(+) T cell survival and memory formation. Nature immunology 15, 1152-1161

73 Hubbard, V.M., et al. (2010) Macroautophagy regulates energy metabolism during effector T cell activation. Journal of immunology 185, 7349-7357 
74 Jia, W., et al. (2015) Autophagy regulates T lymphocyte proliferation through selective degradation of the cell-cycle inhibitor CDKN1B/p27Kip1. Autophagy 11, 2335-2345

75 Pua, H.H., et al. (2007) A critical role for the autophagy gene Atg5 in T cell survival and proliferation. The Journal of experimental medicine 204, 25-31 76 Pua, H.H., et al. (2009) Autophagy is essential for mitochondrial clearance in mature T lymphocytes. Journal of immunology 182, 4046-4055

77 Jia, W. and He, Y.W. (2011) Temporal regulation of intracellular organelle homeostasis in T lymphocytes by autophagy. Journal of immunology 186, 53135322

78 Henson, S.M., et al. (2014) p38 signaling inhibits mTORC1-independent autophagy in senescent human CD8(+) T cells. The Journal of clinical investigation 124, 4004-4016

79 Phadwal, K., et al. (2012) A novel method for autophagy detection in primary cells: impaired levels of macroautophagy in immunosenescent T cells. Autophagy $8,677-689$

80 Eisenberg, T., et al. (2009) Induction of autophagy by spermidine promotes longevity. Nature cell biology 11, 1305-1314

81 Araki, K., et al. (2009) mTOR regulates memory CD8 T-cell differentiation. Nature 460, 108-112

82 Parekh, V.V., et al. (2013) Impaired autophagy, defective T cell homeostasis, and a wasting syndrome in mice with a $\mathrm{T}$ cell-specific deletion of Vps34. Journal of immunology 190, 5086-5101

83 Wei, J., et al. (2016) Autophagy enforces functional integrity of regulatory $\mathrm{T}$ cells by coupling environmental cues and metabolic homeostasis. Nature immunology 17, 277-285

84 Kabat, A.M., et al. (2016) The autophagy gene Atg1611 differentially regulates Treg and TH2 cells to control intestinal inflammation. eLife 5

85 Johnson, K.M., et al. (2002) Aging and developmental transitions in the B cell lineage. International immunology 14, 1313-1323

86 Johnson, S.A., et al. (2002) Aging-dependent exclusion of antigeninexperienced cells from the peripheral B cell repertoire. Journal of immunology 168, 5014-5023

87 Wang, C., et al. (2014) Effects of aging, cytomegalovirus infection, and EBV infection on human B cell repertoires. Journal of immunology 192, 603-611 88 Tabibian-Keissar, H., et al. (2016) Aging affects B-cell antigen receptor repertoire diversity in primary and secondary lymphoid tissues. European journal of immunology 46, 480-492

89 Roukens, A.H., et al. (2011) Elderly subjects have a delayed antibody response and prolonged viraemia following yellow fever vaccination: a prospective controlled cohort study. PloS one 6, e27753

90 Sasaki, S., et al. (2011) Limited efficacy of inactivated influenza vaccine in elderly individuals is associated with decreased production of vaccine-specific antibodies. The Journal of clinical investigation 121, 3109-3119

91 Frasca, D., et al. (2008) Aging down-regulates the transcription factor E2A, activation-induced cytidine deaminase, and Ig class switch in human B cells. Journal of immunology 180, 5283-5290 
92 Frasca, D. and Blomberg, B.B. (2011) Aging impairs murine B cell differentiation and function in primary and secondary lymphoid tissues. Aging and disease 2, 361-373

93 Frasca, D., et al. (2010) Intrinsic defects in B cell response to seasonal influenza vaccination in elderly humans. Vaccine $28,8077-8084$

94 Miller, B.C., et al. (2008) The autophagy gene ATG5 plays an essential role in B lymphocyte development. Autophagy 4, 309-314

95 Pengo, N., et al. (2013) Plasma cells require autophagy for sustainable immunoglobulin production. Nature immunology 14, 298-305

96 Chen, M., et al. (2015) Requirement for autophagy in the long-term persistence but not initial formation of memory B cells. Journal of immunology 194, 2607-2615

97 Hardy, R.R. (2006) B-1 B cells: development, selection, natural autoantibody and leukemia. Current opinion in immunology 18, 547-555

98 Thompson, W.W., et al. (2003) Mortality associated with influenza and respiratory syncytial virus in the United States. Jama 289, 179-186

99 Monto, A.S., et al. (2009) Influenza control in the 21st century: Optimizing protection of older adults. Vaccine 27, 5043-5053

100 Chen, W.H., et al. (2011) Antibody and Th1-type cell-mediated immune responses in elderly and young adults immunized with the standard or a high dose influenza vaccine. Vaccine 29, 2865-2873

$101 \mathrm{Lu}, \mathrm{Q}$., et al. (2016) Homeostatic Control of Innate Lung Inflammation by Vici Syndrome Gene Epg5 and Additional Autophagy Genes Promotes Influenza Pathogenesis. Cell host \& microbe 19, 102-113 102 Hubbard-Lucey, V.M., et al. (2014) Autophagy gene Atg16L1 prevents lethal $\mathrm{T}$ cell alloreactivity mediated by dendritic cells. Immunity $41,579-591$ 103 Grolleau-Julius, A., et al. (2008) Impaired dendritic cell function in aging leads to defective antitumor immunity. Cancer research 68, 6341-6349 104 Vanneman, M. and Dranoff, G. (2012) Combining immunotherapy and targeted therapies in cancer treatment. Nature reviews. Cancer 12, 237-251 105 Ouimet, M., et al. (2011) Autophagy regulates cholesterol efflux from macrophage foam cells via lysosomal acid lipase. Cell metabolism 13, 655-667 106 Chen, W.Q., et al. (2009) Oral rapamycin attenuates inflammation and enhances stability of atherosclerotic plaques in rabbits independent of serum lipid levels. British journal of pharmacology 156, 941-951

107 Hamasaki, M., et al. (2013) Autophagosomes form at ER-mitochondria contact sites. Nature 495, 389-393

108 Lazarou, M., et al. (2015) The ubiquitin kinase PINK1 recruits autophagy receptors to induce mitophagy. Nature 524, 309-314

109 Milan, G., et al. (2015) Regulation of autophagy and the ubiquitinproteasome system by the FoxO transcriptional network during muscle atrophy. Nature communications 6, 6670

110 Settembre, C., et al. (2012) A lysosome-to-nucleus signalling mechanism senses and regulates the lysosome via mTOR and TFEB. The EMBO journal 31, 1095-1108

111 Cheng, C.W., et al. (2014) Prolonged fasting reduces IGF-1/PKA to promote hematopoietic-stem-cell-based regeneration and reverse immunosuppression. Cell stem cell 14, 810-823 
112 Chen, C., et al. (2009) mTOR regulation and therapeutic rejuvenation of aging hematopoietic stem cells. Science signaling 2, ra75

113 Egan, D.F., et al. (2011) Phosphorylation of ULK1 (hATG1) by AMP-activated protein kinase connects energy sensing to mitophagy. Science 331, 456-461 114 Kim, J., et al. (2013) Differential regulation of distinct Vps34 complexes by AMPK in nutrient stress and autophagy. Cell 152, 290-303 115 Salminen, A. and Kaarniranta, K. (2012) AMP-activated protein kinase (AMPK) controls the aging process via an integrated signaling network. Ageing research reviews $11,230-241$

116 Canto, C., et al. (2009) AMPK regulates energy expenditure by modulating NAD+ metabolism and SIRT1 activity. Nature 458, 1056-1060

117 Lee, I.H., et al. (2008) A role for the NAD-dependent deacetylase Sirt1 in the regulation of autophagy. Proceedings of the National Academy of Sciences of the United States of America 105, 3374-3379

118 Price, N.L., et al. (2012) SIRT1 is required for AMPK activation and the beneficial effects of resveratrol on mitochondrial function. Cell metabolism 15, 675-690

119 Satoh, A., et al. (2013) Sirt1 extends life span and delays aging in mice through the regulation of Nk2 homeobox 1 in the DMH and LH. Cell metabolism $18,416-430$

120 Slamova, K., et al. (2015) Adverse effects of AMP-activated protein kinase alpha2-subunit deletion and high-fat diet on heart function and ischemic tolerance in aged female mice. Physiological research / Academia Scientiarum Bohemoslovaca

121 Braidy, N., et al. (2011) Age related changes in NAD+ metabolism oxidative stress and Sirt1 activity in wistar rats. PloS one 6, e19194

122 Hughes, A.L. and Gottschling, D.E. (2012) An early age increase in vacuolar pH limits mitochondrial function and lifespan in yeast. Nature 492, 261-265 123 Sohal, R.S. (1981) Relationship between metabolic rate, lipofuscin accumulation and lysosomal enzyme activity during aging in the adult housefly, Musca domestica. Experimental gerontology 16, 347-355

124 Shamsi, F.A. and Boulton, M. (2001) Inhibition of RPE lysosomal and antioxidant activity by the age pigment lipofuscin. Investigative ophthalmology \& visual science 42, 3041-3046

125 Terman, A., et al. (1999) Ceroid/lipofuscin-loaded human fibroblasts show decreased survival time and diminished autophagocytosis during amino acid starvation. Experimental gerontology 34, 943-957

126 Seidler, S., et al. (2010) Age-dependent alterations of monocyte subsets and monocyte-related chemokine pathways in healthy adults. BMC immunology 11, 30

127 Herrero, C., et al. (2001) IFN-gamma-dependent transcription of MHC class II IA is impaired in macrophages from aged mice. The Journal of clinical investigation 107, 485-493

128 Romao, S., et al. (2013) Autophagy proteins stabilize pathogen-containing phagosomes for prolonged MHC II antigen processing. The Journal of cell biology 203, 757-766

129 Takahashi, R., et al. (2015) Attenuated phagocytosis of secondary necrotic neutrophils by macrophages in aged and SMP30 knockout mice. Geriatrics \& gerontology international 
130 Agrawal, A., et al. (2007) Altered innate immune functioning of dendritic cells in elderly humans: a role of phosphoinositide 3-kinase-signaling pathway. Journal of immunology 178, 6912-6922

131 Lee, H.K., et al. (2010) In vivo requirement for Atg5 in antigen presentation by dendritic cells. Immunity $32,227-239$

132 Reed, M., et al. (2013) Autophagy-inducing protein beclin-1 in dendritic cells regulates CD4 T cell responses and disease severity during respiratory syncytial virus infection. Journal of immunology 191, 2526-2537 


\section{Figure legend}

- Figure 1. Autophagy-controlling pathways. As a stress-response, autophagy is regulated by multiple signals including growth factors, nutrients, energy supply, cytokines, and infections. Certain transcription factors also integrate stress signals to control autophagy. Autophagy has different functions according to its cargo, including quality control (e.g., mitophagy), intracellular pathogen removal (xenophagy), and energy supply/metabolism control (lipophagy). 


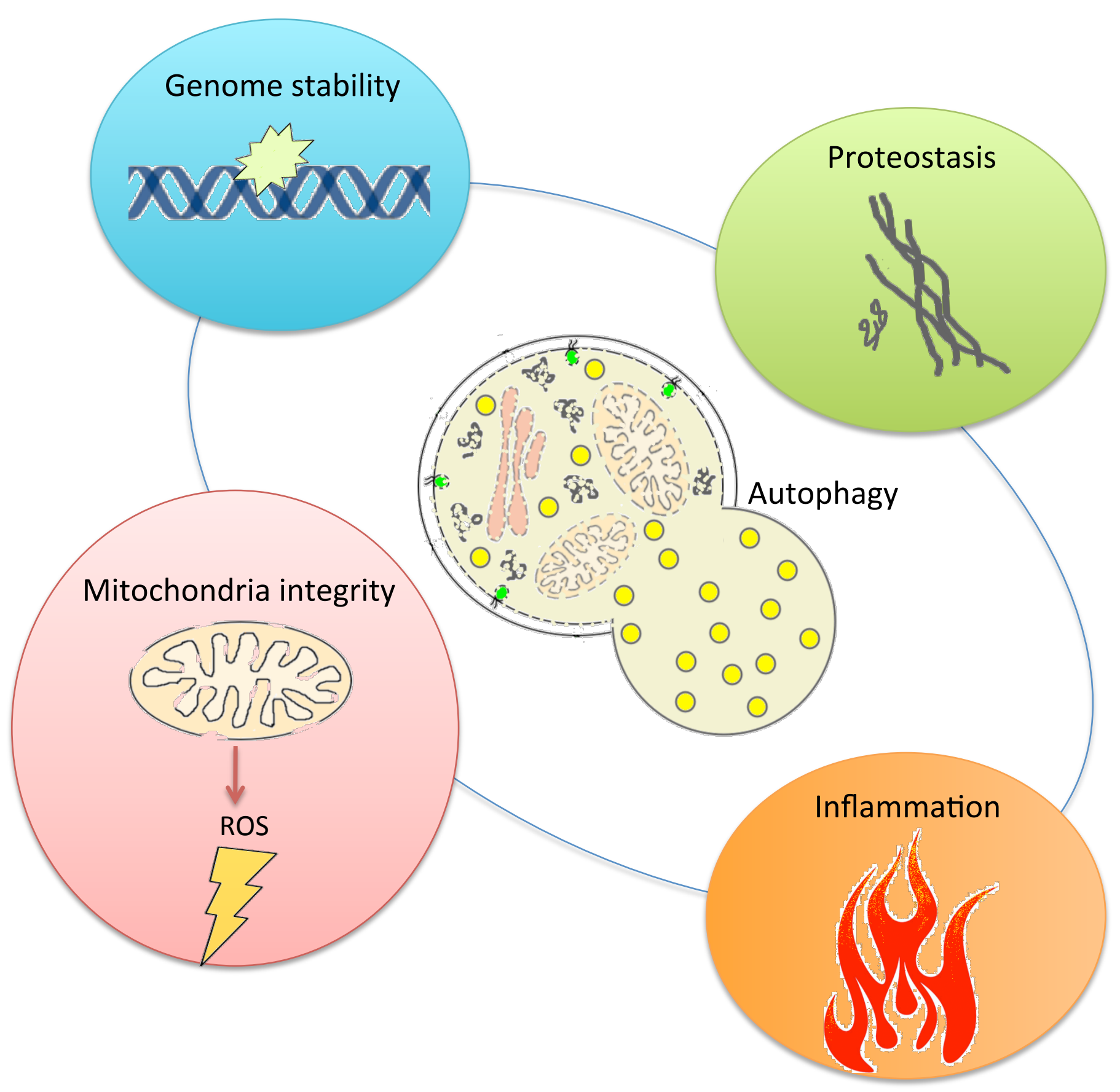

Damaged mitochondria accumulate in aged cells and produce more ROS to

cause oxidative stress. Autophagy

controls mitochondrial health through degradation of damaged mitochondria.

ROS attack single nucleobases causing DNA damage. Autophagy limits ROS accumulation through mitochondrial quality control and thus indirectly maintains genome stability.

In aged mice proteostasis is disrupted and typically large-scale protein aggregation is found. Autophagy degrades protein aggregates and also reduces the formation of protein aggregates by limiting oxidative stress.

The autophagy machinery is used for detection by PAMPs and the secretion of inflammatory cytokines. On the other hand, autophagy degrades the ubiquitinated inflammasome and limits inflammasome activation by clearing intracellular damage and ROS. 


\section{Figure legend}

- Figure 2. Autophagy-Mediated Inhibition of Cellular changes during Senescence. 